\title{
An asymptotic version of the Gowers dichotomy and a new proof of Wagner theorem
}

\author{
Michał Świętek ${ }^{1}$
}

Received: 11 April 2014 / Accepted: 20 October 2015 / Published online: 2 November 2015

(C) The Author(s) 2015. This article is published with open access at Springerlink.com

\begin{abstract}
We present an asymptotic version of the Gowers dichotomy and a new proof of a generalized version of Wagner's theorem. They both concern weaker forms of unconditionality in the context of the asymptotic theory of Banach spaces.
\end{abstract}

Keywords Banach space · Asymptotic theory $\cdot$ Gowers dichotomy

Mathematics Subject Classification 46B20

\section{Introduction}

Probably the most important application of the Gowers theorem is the celebrated Gowers dichotomy, which connects unconditionality and hereditary indecomposability and can be viewed as a dichotomy between the regular and the pathological. On the one hand the existence of an unconditional basis imposes geometric regularity on the unit ball, namely every projection of the unit ball to the space generated by some subset of the basis is contained in a homothety image of the unit ball with some universal constant. In terms of the algebra of operators it implies that the algebra is rich, i.e. all projections to subspaces generated by subsets of the basis and all reflections relative to coordinates are bounded. On the other hand the main geometric consequence of hereditary indecomposability is the zero angle between any two infinite dimensional subspaces. The algebra of operators is almost trivial in this case in the sense that every operator is a singular perturbation of a multiple of the identity.

\footnotetext{
$凶$ Michał Świętek

michal.swietek@uj.edu.pl

1 Faculty of Mathematics and Computer Science, Jagiellonian University,

Łojasiewicza 4, 30-348 Kraków, Poland
} 
The notion of hereditary indecomposability appeared in the infinite dimensional theory of Banach spaces in the long-awaited example of a space without a subspace with an unconditional basis constructed by Maurey and Gowers [1].

One of the ways of understanding how the regularity of the finite dimensional world disappears in the infinite dimensional world is the asymptotic theory of Banach spaces which originated in [2]. Wagner used its notion of asymptotic unconditionality to show the first instance of an asymptotic version of Gowers dichotomy [3]. Then, the asymptotic unconditionality was extended by Tomczak-Jaegremann [4] to the notion of $\alpha$-asymptotic unconditionality which is measured by countable ordinals and concerns not only lengths of sequences of vectors, but also the combinatorial arrangement of supports (with regard to some fixed basis) of their vector components. Using that extended notion Wagner proves in [5] a theorem which captures a moment when regularity is lost because of the lack of an unconditional subspace. We will give a new proof of generalized version of this theorem. Moreover, we will present and prove an asymptotic version of the Gowers dichotomy for the extended notion of asymptotic unconditionality.

Wagner's proof uses the Maurey hierarchy of families of positive integers as a measure of complexity of sequences of vectors occurring in the definition of $\alpha$-asymptotic unconditionality. That family enjoys some necessary properties like increasing degree of complexity (in the sense of the strong Cantor-Bendixson index) and moreover fits very well in Wagner's interesting variation of Gowers' game. Furthermore, in the context of unconditionality the Maurey hierarchy is very convenient in conducting an inductive proof. However, in the proof presented in these notes we will not use transfinite induction nor the Wagner games so we can extract the most important properties of the Maurey hierarchy and base our definition of $\alpha$-asymptotic unconditionality on families with those extracted properties. An example of another family which shares the desired properties is the hierarchy of generalized Schreier families widely used in the theory of Banach spaces. It is worth mentioning that examples of spaces with controlled asymptotic structure are built over the generalized Schreier families [6].

The main ingredient in the proof of Wagner's theorem is an auxiliary quantitative dichotomy (Theorem 4.1) which we will formulate and prove. Its proof is based on the Maurey idea of the proof of the Gowers dichotomy. However, it requires a more delicate argument because of additional structure in the sequences of vectors. In order to control this structure we will introduce a notion of a "combinatorial" angle between subspaces and a special metric on the unit ball, which carry more information than their counterparts in the Maurey proof.

Another ingredient is a certain Ramsey theorem (Theorem 2.4 [7]) that gives information about a relation between different families measuring combinatorial structure of sequences of vectors.

In Sect. 2 we recall or introduce necessary definitions, basic facts and classical tools of the fields of combinatorics and Banach space theory. We also prove some basic properties and lemmas concerning the main notions. After this introduction we pass to Sect. 3 where we discuss Wagner's theorem and the asymptotic dichotomies, and then in section 4 we give all proofs mentioned above.

These notes are based on my master's thesis, which I wrote under the direction of Anna Pelczar-Barwacz and I would like to thank her for her kind introduction to the field, valuable discussions and insightful remarks. 


\section{Basic facts and definitions}

\subsection{Families of finite subsets of the natural numbers}

Let us begin with introducing two basic families of finite subsets of positive integers. In order to do that for every countable ordinal $\beta$ we fix an increasing sequence of ordinals $\left(\beta_{n}\right)_{n \in \mathbb{N}}$ convergent to $\beta$. We keep these sequences fixed throughout these notes. For sets $E, F \in[\mathbb{N}]^{<\infty}$ we write $E<F$ when $\max E<\min F$, where $[\mathbb{N}]^{<\infty}$ denotes the family of finite subsets of natural numbers. Similarly $[\mathbb{N}]$ denotes the family of infinite subsets. We topologize $[\mathbb{N}]^{<\infty}$ and $[\mathbb{N}]$ by the topology of pointwise convergence. We call two subsets $I, J \subset\{1, \ldots, n\}$ a partition of the set $\{1, \ldots, n\}$, if they satisfy $I \cup J=\{1, \ldots, n\}$ and $I \cap J=\emptyset$. We then write $I \sqcup J=\{1, \ldots, n\}$.

We start by defining basic properties of families of positive integers:

Definition 2.1 Let $\mathcal{F}$ be a family of finite subsets of positive integers.

1. $\mathcal{F}$ is hereditary if for every $A \in \mathcal{F}$ and every $B \subset A$ we have $B \in \mathcal{F}$.

2. $\mathcal{F}$ is spreading if for every sequence $\left(n_{1}, \ldots, n_{k}\right) \in \mathcal{F}$ and every sequence $\left(m_{1}, \ldots, m_{k}\right)$ where $m_{i} \geq n_{i}$ for $i=1, \ldots, k$ we have $\left(m_{1}, \ldots, m_{k}\right) \in \mathcal{F}$.

3. $\mathcal{F}$ is compact if it is compact in $[\mathbb{N}]^{<\infty}$.

4. $\mathcal{F}$ is regular if it is hereditary, spreading and compact.

We recall two basic examples of regular families. The first example is the Maurey hierarchy of families which were introduced in [4].

Definition 2.2 The set hierarchy $\left\{\mathcal{M}_{\alpha}\right\}_{\alpha<\omega_{1}}$ is defined inductively.

1. $\mathcal{M}_{0}=\{\emptyset\}$,

2. $\mathcal{M}_{\alpha+1}=\mathcal{M}_{\alpha} \cup\left\{\left(n, n_{1}, \ldots, n_{k}\right) \mid n \in \mathbb{N},\left(n_{1}, \ldots, n_{k}\right) \in \mathcal{M}_{\alpha}, n<n_{1}\right\}$, for every countable ordinal $\alpha$,

3. $\mathcal{M}_{\beta}=\left\{\left(n_{1}, \ldots, n_{k}\right) \mid\left(n_{1}, \ldots, n_{k}\right) \in \mathcal{M}_{\beta_{n}}, n_{1} \geq n, n \in \mathbb{N}\right\}$ for every countable limit ordinal $\beta$.

The second example is the hierarchy of generalized Schreier families. It originated in $[8]$.

Definition 2.3 The set hierarchy $\left\{\mathcal{S}_{\alpha}\right\}_{\alpha<\omega_{1}}$ is defined inductively.

1. $\mathcal{S}_{0}=\{(n) \mid n \in \mathbb{N}\} \cup\{\emptyset\}$,

2. $\mathcal{S}_{\alpha+1}=\left\{\bigcup_{i=1}^{n} F_{i} \mid n \leq F_{1}<\cdots<F_{n}, F_{i} \in \mathcal{S}_{\alpha}\right\}$ for every countable ordinal $\alpha$,

3. $\mathcal{S}_{\beta}=\left\{F \mid F \in \mathcal{S}_{\beta_{n}}, F \geq n, n \in \mathbb{N}\right\}$ for every countable limit ordinal $\beta$.

For every hereditary family $\mathcal{F} \subset[\mathbb{N}]^{<\infty}$ and every set $M \in[\mathbb{N}]$ we have that $\{F \in \mathcal{F} \mid F \subset M\}=\mathcal{F} \cap[M]^{<\infty}$ and we denote this set by $\mathcal{F}[M]$.

A basic combinatorial tool for comparing families is the following

Theorem 2.4 [7] Let $\mathcal{F}, \mathcal{G}$ be hereditary families of finite subsets of positive integers, $N \in[\mathbb{N}]$. Then there exists an infinite subset $M$ of $N$ such that the following dichotomy holds: $\mathcal{F}[M] \subset \mathcal{G}$ or $\mathcal{G}[M] \subset \mathcal{F}$. 
We proceed to the strong Cantor-Bendixson index which will allow us to deduce the correct inclusion. The definition and basic facts come from [9].

Definition 2.5 Let $\mathcal{F} \subset[\mathbb{N}]^{<\infty}$ be a regular family. First we define the derivative set of $\mathcal{F}$ namely $D(\mathcal{F})=\{F \in \mathcal{F} \mid F$ is a cluster point in $\mathcal{F}\}$. Then we continue inductively. For every countable ordinal $\alpha$ we set $D^{\alpha+1}(\mathcal{F})=D\left(D^{\alpha}(\mathcal{F})\right)$ and for every countable limit ordinal $\beta$ we set $D^{\beta}(\mathcal{F})=\bigcap_{\alpha<\beta} D^{\alpha}(\mathcal{F})$. For convenience we write $D^{0}(\mathcal{F})=\mathcal{F}$. Finally we define the strong Cantor-Bendixson index of the family $\mathcal{F}$ to be the ordinal

$$
I(\mathcal{F})=\min \left\{\alpha<\omega_{1} \mid D^{\alpha}=\{\emptyset\}\right\}
$$

Remark 2.6 The usual Cantor-Bendixson index of $\mathcal{F}$ is equal to $I(\mathcal{F})+1$. This is due to the fact that the set $\{\varnothing\}$ represents the null function and it is necessary to take one more derivative to obtain $\varnothing$.

The complexities of our basic families are as follows

Proposition 2.7 For each countable ordinal $\alpha$ we have the following:

1. $I\left(\mathcal{M}_{\alpha}\right)=\alpha$,

2. $I\left(\mathcal{S}_{\alpha}\right)=\omega^{\alpha}$.

Moreover, the index remains constant when passing to subsets, i.e. for every infinite set $M$ we have:

1. $I\left(\mathcal{M}_{\alpha}[M]\right)=\alpha$,

2. $I\left(\mathcal{S}_{\alpha}[M]\right)=\omega^{\alpha}$.

\subsection{Basic Banach space notions: admissible families}

From now on we assume that every Banach space is infinite dimensional. Let $X$ be a Banach space and recall that a sequence $\left(e_{n}\right)_{n \in \mathbb{N}}$ is called (a Schauder) basis, if every vector $x$ in $X$ has a unique expansion in a series $\sum a_{n} e_{n}$ for some sequence of scalars $\left(a_{n}\right)_{n \in \mathbb{N}}$. A support of a non zero vector $x=\sum a_{n} e_{n}$ is the set $\operatorname{supp}_{X} x=\{n \in$ $\left.\mathbb{N} \mid a_{n} \neq 0\right\}$. A sequence $\left(x_{n}\right)_{n}$ is called a block (basic) sequence, if the supports of its elements are finite consecutive sets. The set of finite block sequences with norm less than or equal to one is denoted by $\Sigma(X)$. We say that a subspace is a block subspace, if it is generated by some block sequence. The span of $\left(x_{n}\right)_{n}$ is denoted by $\left\langle x_{n} \mid n\right\rangle$.

A basis $\left(e_{n}\right)$ is called $C$-unconditional, $C \geq 1$, if for every sequence of signs $\left(\varepsilon_{n}\right)$ the operator $\sum a_{n} e_{n} \mapsto \sum \varepsilon_{n} a_{n} e_{n}$ has norm bounded above by $C$.

Now we will define admissible families - a notion which we will use as a measure of complexity of block sequences. From now up to the end of the paper we fix a hierarchy $\left\{\mathcal{F}_{\alpha}\right\}_{\alpha<\omega_{1}}$ of families of finite sets of the natural numbers such that the following conditions are satisfied:

$(\mathcal{F} 1)$ the family $\mathcal{F}_{\alpha}$ is regular for every countable ordinal $\alpha$,

$(\mathcal{F} 2)$ the families $\left\{\mathcal{F}_{\alpha}\right\}_{\alpha<\omega_{1}}$ satisfy the diagonal construction condition, i.e. for every countable limit $\beta$ we have 


$$
\mathcal{F}_{\beta}=\left\{F \mid F \in \mathcal{F}_{\beta_{n}}, n \leq F, n \in \mathbb{N}\right\}
$$

$(\mathcal{F} 3)$ the complexity of the family $\mathcal{F}_{\alpha}$ increases with $\alpha$, i.e. we have

$$
\alpha_{1}<\alpha_{2} \Rightarrow I\left(\mathcal{F}_{\alpha_{1}}\right)<I\left(\mathcal{F}_{\alpha_{2}}\right)
$$

Remark 2.8 The condition $(\mathcal{F} 1)$ implies that the index of family $\mathcal{F}_{\alpha}$ is constant when passing to subspaces:

$$
I\left(\mathcal{F}_{\alpha}[M]\right)=I\left(\mathcal{F}_{\alpha}\right) \text { for every infinite subset } M \text { of } \mathbb{N} \text {. }
$$

We see that the diagonal construction condition $(\mathcal{F} 2)$ depends on the choice of converging sequences and that is why we fix them. A particular choice is irrelevant and does not have any influence on statements of definitions and theorems.

Definition 2.9 Let $X$ be a Banach space with a basis $\left(e_{n}\right)_{n \in \mathbb{N}}$, fix a hierarchy $\left\{\mathcal{F}_{\alpha}\right\}$ satisfying the conditions $(\mathcal{F} 1)-(\mathcal{F} 3)$ and fix an ordinal $\alpha$. A sequence $\left(v_{i}\right)_{i=1}^{k}$ is called $\mathcal{F}_{\alpha}$-admissible if the corresponding sequence $\left(\min \left(\operatorname{supp}_{X} v_{i}\right)\right)_{i=0}^{k}$ is a member of the family $\mathcal{F}_{\alpha}$. The set of $\mathcal{F}_{\alpha}$-admissible block sequences is denoted by $\Sigma_{\mathcal{F}_{\alpha}}(X)$.

The concept of admissible families comes from [10].

Remark 2.10 The set $\Sigma_{\mathcal{F}_{\alpha}}(X)$ of $\mathcal{F}_{\alpha}$-admissible sequences depends on the choice of a basis of $X$. In the following every space will always be considered with one fixed basis, but we have to be cautious when dealing with subspaces because of the fact that it is not true in general that $\Sigma_{\mathcal{F}_{\alpha}}(Y)=\Sigma_{\mathcal{F}_{\alpha}}(X) \cap \Sigma(Y)$. We see that if $Y$ is an infinite dimensional block subspace of $X$, then we have $\Sigma_{\mathcal{F}_{\alpha}}(Y) \subset \Sigma_{\mathcal{F}_{\alpha}}(X)$.

\section{3 $\mathcal{F}_{\alpha}$-unconditionality and $H I_{\mathcal{F}_{\alpha}}$ property}

In this subsection we define central objects of interest: $\mathcal{F}_{\alpha}$-unconditionality and the $H I_{\mathcal{F}_{\alpha}}$ property. But before that we recall the following definition: A Banach space is called hereditarily indecomposable ( $H$ I for short), if no infinite dimensional subspace is a direct sum of two closed infinite dimensional subspaces. To make the above definition more geometric we introduce some notion of an angle.

Definition 2.11 Let $X$ be a Banach space and let $U, V$ be subspaces of $X$ of arbitrary dimension. We define the angle $a$ between subspaces $U, V$ as

$$
a(U, V)=\inf \{\|u-v\| \mid u \in S(U), v \in S(V)\} .
$$

Proposition 2.12 [11] Let $X$ be an infinite dimensional Banach space. Then the following conditions are equivalent:

1. the space $X$ is $H I$,

2. for all infinite dimensional subspaces $U, V$ the angle $a(U, V)$ equals 0 . 
The above notion of angle is convenient with respect to the $H I$ property, and now we define another one which is better to work with unconditionality.

Definition 2.13 Let $X$ be a Banach space and let $U, V$ be subspaces of $X$ of arbitrary dimension. We define the angle $b$ between the subspaces $U, V$ as

$$
b(U, V)=\min \{\inf \{\operatorname{dist}(u, S(V)) \mid u \in B(U)\}, \inf \{\operatorname{dist}(v, S(U)) \mid v \in B(V)\}\} .
$$

We see that the angles $a$ and $b$ are equivalent in the following sense:

$$
b(U, V) \leq a(U, V) \leq 2 b(U, V), \quad \text { for every subspaces } U, V .
$$

Concerning unconditionality in terms of angles we have

Proposition 2.14 [11] Let $\left(e_{n}\right)_{n=0}^{m}$ be a sequence of vectors in $X$.

1. If the sequence $\left(e_{n}\right)_{n=0}^{m}$ is $K$-unconditional, $K \geq 1$, then for every partition $I \sqcup J=\{0, \ldots, m\}$ we have $b\left(\left\langle e_{i} \mid i \in I\right\rangle,\left\langle e_{j} \mid j \in J\right\rangle\right) \geq \frac{2}{K+1}$.

2. If for some fixed $\varepsilon>0$ and every partition $I \sqcup J=\{0, \ldots, m\}$ it is true that $b\left(\left\langle e_{i} \mid i \in I\right\rangle,\left\langle e_{j} \mid j \in J\right\rangle\right) \geq \varepsilon$ then the sequence $\left(e_{n}\right)_{n=0}^{m}$ is $\frac{2}{\varepsilon}$-unconditional.

Now let us introduce a notion which is an idea of building a bridge between unconditionality of finite and infinite dimensional world.

Definition 2.15 Let $\alpha$ be a countable ordinal. If $X$ is a Banach space with a basis and $Y$ is an infinite dimensional block subspace of $X$, then we say that $Y$ is $\mathcal{F}_{\alpha^{-}}$ asymptotically unconditional with a constant $C$, if the set of $\mathcal{F}_{\alpha}$-admissible sequences $\Sigma_{\mathcal{F}_{\alpha}}(Y)$ consists of $C$-unconditional sequences. The least such constant $C$ is called an $\mathcal{F}_{\alpha}$-asymptotically unconditional constant.

The notion of asymptotic unconditionality appeared for the first time in [12] and coincides with our $\omega$-asymptotic unconditionality for the Maurey hierarchy. It was also used in [2], but in a general form it was used in [4] as a tool in showing that a space with bounded distortion contains an unconditional sequence. The following theorem is also contained in [4].

Theorem 2.16 Let $X$ be a Banach space with a basis. If $X$ contains an $\mathcal{F}_{\alpha^{-}}$ asymptotically unconditional subspace for every $\alpha<\omega_{1}$, then $X$ contains an unconditional basic sequence.

The notion of $\mathcal{F}_{\alpha}$-asymptotic unconditionality is much weaker than ordinary unconditionality, so as its "opposite" we consider a property which is more regular than the HI property.

Definition 2.17 Let $X$ be a Banach space with a basis, let $Y$ be an infinite dimensional block subspace of $X$ and fix $\alpha<\omega_{1}$ and $\varepsilon>0$.

1. The subspace $Y$ is called $H I_{\mathcal{F}_{\alpha}, X}(\varepsilon)$, if for all infinite dimensional subspaces $U_{0}, U_{1}$ of $Y$ there exists sequence $\left(u_{i}\right)_{i=0}^{K} \in \Sigma_{\mathcal{F}_{\alpha}}(X)$ such that the following condition holds:

$$
\left(u_{2 i+j}\right)_{i} \in \Sigma\left(U_{j}\right), \quad j=0,1 \quad \text { and } \quad b\left(\left\langle u_{2 i} \mid 2 i \leq K\right\rangle,\left\langle u_{2 i+1} \mid 2 i+1 \leq K\right\rangle\right) \leq \varepsilon .
$$


2. The subspace $Y$ is called $H I_{\mathcal{F}_{\alpha}, X}$, if it is $H I_{\mathcal{F}_{\alpha}, X}\left(\varepsilon^{\prime}\right)$ for every $\varepsilon^{\prime}>0$.

3. The subspace $Y$ is called $H I_{\mathcal{F}_{\alpha}}(\varepsilon)$, if it is $H I_{\mathcal{F}_{\alpha}, Y}(\varepsilon)$.

4. The subspace $Y$ is called $H I_{\mathcal{F}_{\alpha}}$, if it is $H I_{\mathcal{F}_{\alpha}}\left(\varepsilon^{\prime}\right)$ for every $\varepsilon^{\prime}>0$.

Examples for the Maurey hierarchy:

1. The space constructed by Maurey and Gowers [1] was the first example of an $H I$ space and therefore the first example of a space without an unconditional sequence. Moreover it is not $\mathcal{M}_{\alpha}$-asymptotically unconditional for any $\alpha \geq \omega$. Let us notice that every infinite dimensional Banach space is $\mathcal{M}_{n}$-asymptotically unconditional, hence Gowers-Maurey's space has the worst possible asymptotic unconditional structure.

2. Gowers has constructed in [13] a space which is $H I$ so does not contain any unconditional sequence but is additionally $\mathcal{M}_{\omega}$-asymptotically unconditional.

3. Another fundamental example is a space constructed by Argyros and Deliyanni [10]. That space is also $H I$ and $\mathcal{M}_{\omega}$-asymptotically unconditional, and moreover it is asymptotic- $\ell_{1}$.

\section{Some Banach space dichotomies}

\subsection{Wagner's theorem}

In [5] Wagner used transfinite induction to obtain a new proof of the so called Gowers combinatorial lemma [14] as well as the following

Theorem 3.1 (Wagner [5]) Let X be a Banach space with a basis and without an unconditional sequence. Then there exist a block subspace $Y$ of $X$, a countable ordinal $\beta$ and a function $\alpha(\varepsilon)$ which is increasingly converging to $\beta$ with $\varepsilon$ converging to 0 such that the following conditions hold:

1. $\beta$ is the least ordinal $\gamma$ such that the subspace $Y$ does not contain any $\mathcal{M}_{\gamma^{-}}$ asymptotically unconditional subspace,

2. the subspace $Y$ is $H I_{\mathcal{M}_{\alpha(\varepsilon)}}(\varepsilon)$ for every $\varepsilon>0$.

Later in the paper we will give a new proof of Wagner's theorem for an abstract family $\left\{\mathcal{F}_{\alpha}\right\}_{\alpha<\omega_{1}}$ satisfying conditions $(\mathcal{F} 1)-(\mathcal{F} 3)$. What we are going to prove is

Theorem 3.2 (Wagner [version] $\mathcal{F}$ ]) Let X be a Banach space with a basis and without an unconditional sequence, fix an arbitrary family $\left\{\mathcal{F}_{\alpha}\right\}_{\alpha<\omega_{1}}$ satisfying conditions $(\mathcal{F} 1)-(\mathcal{F} 3)$ and suppose that the ordinal $\beta=\min \{\beta(Z) \mid Z \subset X\}$, where

$$
\beta(Z)=\min \left\{\gamma<\omega_{1} \mid \neg\left(\exists W \subset Z: W \text { is } \mathcal{F}_{\gamma} \text {-asymptotically unconditional }\right)\right\}
$$

is a countable limit ordinal.

Then there exists a sequence $\left(\alpha_{n}\right)_{n \in \mathbb{N}}$ increasingly converging to $\beta$ and there exists a subspace $Y$ of $X$ such that the following conditions hold:

1. $\beta$ is the least ordinal $\gamma$ such that the subspace $Y$ does not any contain $\mathcal{F}_{\gamma^{-}}$ asymptotically unconditional subspace,

2. the subspace $Y$ is $H I_{\mathcal{F}_{\alpha_{n}}}\left(\frac{1}{n}\right)$ for every positive integer $n$. 
Remark 3.3 1. We see that Theorem 2.16 implies that the ordinal $\beta$ in the above theorem is well defined.

2. For the Maurey hierarchy the assumption that $\beta$ is a limit ordinal follows from its definition.

3. For the Maurey hierarchy the above theorem is a reformulation of Wagner's theorem.

The main tools in the proof of Wagner's theorem [version $\mathcal{F}$ ] are Gasparis' dichotomy and the quantified version of $\mathcal{F}_{\alpha}$-dichotomy which is described in the next section.

\subsection{Dichotomies for $\mathcal{F}_{\alpha}$-admissible families}

Using tools constructed in the next section we will prove the following dichotomy for the arbitrary hierarchy $\left\{\mathcal{F}_{\alpha}\right\}_{\alpha<\omega_{1}}$ of families satisfying conditions $(\mathcal{F} 1)-(\mathcal{F} 3)$.

Theorem 3.4 ( $\mathcal{F}_{\alpha}$-dichotomy) Let $X$ be a Banach space without an unconditional sequence, let $\alpha$ be a countable ordinal. Then there exists a subspace $Y$ of $X$ with a basis such that one of the following conditions holds:

1. the subspace $Y$ is $\mathcal{F}_{\alpha}$-asymptotically unconditional,

2. the subspace $Y$ is $H I_{\mathcal{F}_{\alpha+1}}$.

Subsequently using Wagner's theorem [version $\mathcal{F}$ ] (Theorem 3.2) we will strengthen the above theorem for limit ordinals in a way that in the second possibility there is the $H I_{\mathcal{F}_{\alpha}}$ property.

\section{Proofs of the main results}

We start this section with a quantified version of the $\mathcal{F}_{\alpha}$-dichotomy which will allow us to give a new proof of Wagner's theorem in a generalized version. In its proof we follow Maurey's proof of the Gowers dichotomy [11], but with additional care arising from the need of controlling supports of blocks. Because of the nature of our theorems using the Mazur theorem we may assume that the starting space has a basis and moreover we can assume that it is bimonotone. Furthermore using the classical Bessaga-Peczyski theorem we can restrict ourselves to block subspaces. Let us also recall that we have fixed a hierarchy $\left\{\mathcal{F}_{\alpha}\right\}_{\alpha<\omega_{1}}$ satisfying conditions $(\mathcal{F} 1)-(\mathcal{F} 3)$.

\subsection{The quantified version of the $\mathcal{F}_{\alpha}$-dichotomy}

The purpose of this subsection is to prove the following

Theorem 4.1 (Quantified $\mathcal{F}_{\alpha}$-dichotomy) Let X be a Banach space with a basis and without an unconditional sequence, let $\alpha$ be a countable ordinal and let $\varepsilon>0$. Then there exists a subspace $Y$ of $X$ such that one of the following conditions holds:

1. the subspace $Y$ is $\mathcal{F}_{\alpha}$-asymptotically unconditional with constant $\frac{4}{\varepsilon}$,

2. the subspace $Y$ is $H I_{\mathcal{F}_{\alpha}, X}(\varepsilon)$. 
We need some preparatory work. Let us begin with a definition of a notion of angle between subspaces, which will assist our proof of the quantified $\mathcal{F}_{\alpha}$-dichotomy.

Definition 4.2 Let $X$ be a Banach space with a basis. Then for arbitrary subspaces $U, V$ we define the combinatorial angle $k_{\mathcal{F}_{\alpha}} \in[0,2]$ as:

$$
\begin{aligned}
& k_{\mathcal{F}_{\alpha}}(U, V)= \inf \left\{a\left(\left\langle x_{i} \mid i \in I\right\rangle,\left\langle x_{j} \mid j \in J\right\rangle\right) \mid\left(x_{i}\right)_{i=1}^{K} \in \Sigma_{\mathcal{F}_{\alpha}}(X),\right. \\
&\left.I \sqcup J=\{1, \ldots, K\}:\left(x_{i}\right)_{i \in I} \in \Sigma(U),\left(x_{j}\right)_{j \in J} \in \Sigma(V)\right\} .
\end{aligned}
$$

We define a new metric on the unit ball which is a modification of the standard metric determined by a norm. That modification is necessary because of the definition of $\mathcal{F}_{\alpha}$-admissibility which demands care with supports of blocks.

Definition 4.3 For a Banach space $X$ we define a metric $d$ on the unit ball $B(X)$ in the following way:

$$
d(x, y)= \begin{cases}\|x-y\|, & \text { if } \operatorname{supp}_{X} x=\operatorname{supp}_{X} y \\ 1, & \text { otherwise. }\end{cases}
$$

Based on the metric $d$ we define a distance between finite dimensional subspaces of the space $X$ as Hausdorff distance $d_{H}$ on unit balls. We denote the space of finite dimensional block subspaces of the space $X$ endowed with the metric $d_{H}$ by $\mathcal{X}$. We have the following simple observation

Observation 4.4 If $E, F$ are in $\mathcal{X}$ and $d_{H}(E, F)<1$, then $\operatorname{dim} E=\operatorname{dim} F$ and, if sequences of blocks $\left(e_{1}, \ldots, e_{n}\right),\left(f_{1}, \ldots, f_{m}\right)$ are bases of $E$ and $F$ respectively, then $n=m$ and $\operatorname{supp}_{X}\left(e_{i}\right)=\operatorname{supp}_{X}\left(f_{i}\right)$, for $i=1, \ldots, n$.

Let us see that for $E=F$ the above observation implies that finite dimensional block subspaces have uniquely determined supports of bases.

Now we will prove an approximation lemma which will allow us to carry some inductive countable construction for a countable dense subset of the space $\mathcal{X}$ to the whole space $\mathcal{X}$. The statement of the lemma is similar to Maurey's lemma 1 [11] but has a different proof due to the required $\mathcal{F}_{\alpha}$-admissibility.

Lemma 4.5 Let $X$ be a Banach space with a basis, let $M, Z$ be infinite dimensional block subspaces of $X$, let $E, E^{\prime}$ be finite dimensional block subspaces. If $d_{H}\left(E, E^{\prime}\right)<$ 1 then the following estimate holds:

$$
\sup _{U \subset Z} k \mathcal{F}_{\alpha}\left(E^{\prime}+U, M\right) \leq \sup _{V \subset Z} k_{\mathcal{F}_{\alpha}}(E+V, M)+2 d_{H}\left(E^{\prime}, E\right) .
$$

Proof Let us fix a number $s>\sup _{V \subset Z} k_{\mathcal{F}_{\alpha}}(E+V, M)$, a subspace $U \subset Z$ and let us denote $d=d_{H}\left(E^{\prime}, E\right)$. Then, take an infinite dimensional subspace $U^{\prime} \subset U$ satisfying the following condition: $\operatorname{supp}_{X}(E)=\operatorname{supp}_{X}\left(E^{\prime}\right)<\operatorname{supp}_{X}\left(U^{\prime}\right)$. Then we have $k_{\mathcal{F}_{\alpha}}\left(E+U^{\prime}, M\right)<s$ so there exist a sequence $\left(x_{i}\right)_{i=1}^{K} \in \Sigma_{\mathcal{F}_{\alpha}}(X)$ and a partition $I \sqcup J=\{1, \ldots, K\}$ such that $\left(x_{i}\right)_{i \in I} \in \Sigma(E+U),\left(x_{j}\right)_{j \in J} \in \Sigma(M)$ and 
$\mathrm{a}\left(\left\langle x_{i} \mid i \in I\right\rangle,\left\langle x_{j} \mid j \in J\right\rangle\right)<s$. Therefore there exist vectors $e \in E, u^{\prime} \in U^{\prime}$ such that $e+u^{\prime} \in S\left(\left\langle x_{i} \mid i \in I\right\rangle\right)$ and $m \in S\left(\left\langle x_{j} \mid j \in J\right\rangle\right)$ which satisfy $\left\|e+u^{\prime}-m\right\|<s$.

The fact that the basis of $X$ is bimonotone gives $\|e\| \leq\left\|e+u^{\prime}\right\|=1$ hence there is a vector $e^{\prime} \in B\left(E^{\prime}\right)$ such that $\left\|e-e^{\prime}\right\| \leq d$ and $\operatorname{supp}_{X}\left(e^{\prime}\right)=\operatorname{supp}_{X}(e)$. Now we have $1-d \leq\left\|e^{\prime}+u^{\prime}\right\| \leq 1+d$ which for $x^{\prime}=\frac{e^{\prime}+u^{\prime}}{\left\|e^{\prime}+u^{\prime}\right\|} \in S\left(E^{\prime}+U^{\prime}\right)$ implies that $\left\|x^{\prime}-\left(e^{\prime}+u^{\prime}\right)\right\| \leq d$, and this gives $\left\|x^{\prime}-m\right\| \leq\left\|x^{\prime}-\left(e^{\prime}+u^{\prime}\right)\right\|+\left\|e^{\prime}-e\right\|+\left\|e+u^{\prime}-m\right\| \leq$ $s+2 d$. By now we have vectors $x^{\prime} \in S\left(E^{\prime}+U^{\prime}\right), m \in S(M)$ which are close. Next we we will show that they are of appropriate form.

We construct a block sequence $\left(x_{i}^{\prime}\right)_{i \in I^{\prime}} \in \Sigma\left(E^{\prime}+U\right)$ such that the sequence consisting of elements of the sequences $\left(x_{i}^{\prime}\right)_{i \in I^{\prime}}$ and $\left(x_{j}\right)_{j \in J}$ is $\mathcal{F}_{\alpha}$-admissible and $x^{\prime} \in S\left(\left\langle x_{i}^{\prime} \mid i \in I^{\prime}\right\rangle\right)$.

Because of the inequality $\operatorname{supp}_{X}(E)<\operatorname{supp}_{X}\left(U^{\prime}\right)$ we can represent the vector $e+u^{\prime}$ in terms of the basis either in the form

$$
\begin{aligned}
& e+u^{\prime}=\sum_{i \in I_{E}} a_{i} x_{i}+\sum_{i \in I_{U^{\prime}}} a_{i} x_{i}, \quad \text { or } \\
& e+u^{\prime}=\sum_{i \in I_{E}} a_{i} x_{i}+a_{i 0} x_{i_{0}}+\sum_{i \in I_{U^{\prime}}} a_{i} x_{i} .
\end{aligned}
$$

The first sum is an element of $E$, the index $i_{0}$ represents at most one element for which $\operatorname{supp}_{X}\left(x_{i_{0}}\right) \cap \operatorname{supp}_{X}(E) \neq \varnothing$ and $\operatorname{supp}_{X}\left(x_{i_{0}}\right) \cap \operatorname{supp}_{X}\left(U^{\prime}\right) \neq \varnothing$ and the second sum is an element of $U^{\prime}$. If the index $i_{0}$ exists, then let us define elements $x(E), x\left(U^{\prime}\right)$ as parts of the vector $a_{i_{0}} x_{i_{0}}$ belonging to spaces $E, U^{\prime}$ respectively. If it doesn't exist then we set $x(E)=0, x\left(U^{\prime}\right)=0$.

From our assumptions and Observation 4.4 we know that the spaces $E, E^{\prime}$ have equal dimensions and bases with uniquely determined supports with respect to $X$. Therefore we can group basis vectors of $E^{\prime}$ which appear in $e^{\prime}$ in the same manner in which respective basic vectors of $E$ are grouped in the expansion $e=\sum_{i \in I_{E}} a_{i} x_{i}+$ $x(E)$.

According to this grouping we obtain the block sequence $\left(y_{i}\right)_{i \in I_{E}}$ in $E^{\prime}$ and the vector $y\left(E^{\prime}\right) \in E^{\prime}$ for which the following conditions hold:

(i) $\operatorname{supp}_{X}\left(y_{i}\right)=\operatorname{supp}_{X}\left(x_{i}\right)$ for $i \in I_{E}$,

(ii) $\operatorname{supp}_{X}\left(y\left(E^{\prime}\right)\right)=\operatorname{supp}_{X}(x(E))$,

(iii) $e^{\prime}=\sum_{i \in I_{E}} b_{i} y_{i}+y\left(E^{\prime}\right)$ for some scalars $\left(b_{i}\right)$.

Let $\left(x_{i}^{\prime}\right)_{i \in I^{\prime}}$ be a block sequence consisting of elements of the sequences $\left(y_{i}\right)_{i \in I_{E}}$ and $\left(x_{i}\right)_{i \in I_{U^{\prime}}}$, if $y\left(E^{\prime}\right)+x\left(U^{\prime}\right)=0$, or of those elements and the vector $y\left(E^{\prime}\right)+x\left(U^{\prime}\right)$, if $y\left(E^{\prime}\right)+x\left(U^{\prime}\right) \neq 0$. Conditions (i) and (ii) imply that the block sequence consisting of elements of the sequences $\left(x_{i}^{\prime}\right)_{i \in I^{\prime}}$ and $\left(x_{j}\right)_{j \in J}$ is $\mathcal{F}_{\alpha}$-admissible. Furthermore we have $x^{\prime} \in S\left(\left\langle x_{i}^{\prime} \mid i \in I^{\prime}\right\rangle\right)$, thus finally:

$$
k_{\mathcal{F}_{\alpha}}\left(E^{\prime}+U, M\right) \leq k_{\mathcal{F}_{\alpha}}\left(E^{\prime}+U^{\prime}, M\right) \leq a\left(\left\langle x_{i}^{\prime} \mid i \in I^{\prime}\right\rangle,\left\langle x_{j} \mid j \in J\right\rangle\right) \leq s+2 d .
$$

And the proof of the lemma is finished.

Now we can pass to the proof of the Theorem 4.1 following the Maurey scheme. 
Proof of the Theorem 4.1 For a pair of finite dimensional block subspaces $(E, F)$ of $X$ and an infinite dimensional block subspace $Z$ we define

$$
K_{\mathcal{F}_{\alpha}}(E, F, Z)=\sup _{U, V \subset Z} k_{\mathcal{F}_{\alpha}}(E+U, F+V) .
$$

We see that the number $K_{\mathcal{F}_{\alpha}}(E, F, Z)$ is finite for every $(E, F, Z)$. Fix $\varepsilon>0$. Let us introduce terminology inspired by [11] whose roots reach into the proof of the generalization of the Nash-Williams theorem in [15].

From now on our proof of the quantified version of $\mathcal{F}_{\alpha}$-dichotomy is almost identical to Maurey's in [11], so we will only indicate minor differences between them.

We say that a pair of finite dimensional subspaces $(E, F)$ accepts an infinite dimensional subspace $Z$, if $K_{\mathcal{F}_{\alpha}}(E, F, Z)<\varepsilon$. We say that a pair $(E, F)$ rejects an infinite dimensional subspace $Z$, if no subspace $Z^{\prime} \subset Z$ is accepted by the pair $(E, F)$. Note the following easy observation

Observation 4.6 1. If the pair $(\{0\},\{0\})$ accepts a subspace $Z$ then $Z$ has the $H I_{\mathcal{F}_{\alpha}, X}(\varepsilon)$ property.

2. If a pair $(E, F)$ rejects a subspace $Z$ then $k_{\mathcal{F}_{\alpha}}(E, F) \geq \varepsilon$.

3. For every pair $(E, F)$ and every finite dimensional subspace $G$ we have

$$
K_{\mathcal{F}_{\alpha}}(E, F, Z+G)=K_{\mathcal{F}_{\alpha}}(E, F, Z)
$$

Having the approximation lemma and the above observation we can continue as Maurey in his proof of the Gowers dichotomy [11] (compare Claims 1, 2 and Lemma 2 ). It is easily seen that the set $\mathcal{D}$ consisting of the finite dimensional block subspaces spanned by blocks with rational coordinates is dense in the space $\left(\mathcal{X}, d_{H}\right)$. Similarly to [11] we can obtain the following

Observation 4.7 There exists an infinite dimensional subspace $Z_{0}$ of $X$ such that for every pair $(E, F)$, where $E, F \in \mathcal{D}$ and every rational number $q \in(0, \varepsilon)$ either $K_{\mathcal{F}_{\alpha}}(E, F, Z)<q$ or for every infinite dimensional subspace $Z^{\prime} \subset Z_{0}$ we have $K_{\mathcal{F}_{\alpha}}\left(E, F, Z^{\prime}\right) \geq q$.

Then using Lemma 4.5 we can transfer the above observation to an arbitrary pair.

Observation 4.8 For every pair $(E, F)$, where $E, F \in \mathcal{X}$, either $(E, F)$ accepts $Z_{0}$ or $(E, F)$ rejects $Z_{0}$.

Having the stabilizing subspace $Z_{0}$ we can now come back to the main proof. If the pair ( $\{0\},\{0\})$ accepts the space $Z_{0}$, then according to Observation 4.6.(1) the space $Z_{0}$ is $H I_{\mathcal{F}_{\alpha}, X}(\varepsilon)$ and condition (1) of Theorem 4.1 holds. If it does not accept the space $Z_{0}$ then we construct a block sequence $\left(z_{n}\right)_{n \in \mathbb{N}}$ such that the space $Y=\left\langle z_{n} \mid n \in \mathbb{N}\right\rangle$ is $\mathcal{F}_{\alpha}$-asymptotically unconditional with constant $4 / \varepsilon$, i.e. $Y$ will satisfy condition (2) of Theorem 4.1.

The proofs of the next lemma and corollaries are analogous to those in [11].

Lemma 4.9 If a pair $(E, F)$ rejects $Z_{0}$, then in every infinite dimensional block subspace $Z^{\prime} \subset Z_{0}$ there exists an infinite dimensional block subspace $U^{\prime} \subset Z^{\prime}$ such that for every finite dimensional subspace $E^{\prime}$ in $U^{\prime}$ the pair $\left(E+E^{\prime}, F\right)$ rejects $Z_{0}$. 
By an easy induction argument we obtain the following:

Corollary 4.10 If $\left(E_{a}, F_{a}\right)_{a \in A}$ is a finite family of pairs and every pair $\left(E_{a}, F_{a}\right)$ rejects $Z_{0}$, then in every infinite dimensional block subspace $Z^{\prime}$ of $Z_{0}$ there exists a normalized block $z \in Z^{\prime}$ such that the pair $\left(E_{a}+\langle z\rangle, F_{a}\right)$ rejects $Z_{0}$, for $a \in A$.

Now we can finish the proof by constructing inductively an infinite block sequence $\left(z_{n}\right)_{n \in \mathbb{N}}$ such that for every $n$ the following is true:

(*) for every partition $I \sqcup J=\{1, \ldots, n\}$ the pair $\left(E_{I}, E_{J}\right)$ rejects $Z_{0}$, where $E_{K}$ denotes the set $\left\langle z_{k} \mid k \in K\right\rangle$.

Take an arbitrary vector $z_{1}$. Then suppose we have constructed a block sequence $\left(z_{1}, \ldots, z_{n}\right)$ satisfying $(*)$. Let $A_{n}$ be the set of indices such that the family $\left(E_{a}, F_{a}\right)_{a \in A_{n}}$ consists of pairs $\left(E_{I}, E_{J}\right)$, for every possible partition $I \sqcup J=$ $\{1, \ldots, n\}$. Then take an infinite dimensional subspace $Z^{\prime}$ of $Z_{0}$ such that $\operatorname{supp}_{X}\left(Z^{\prime}\right)$ begins after $\operatorname{supp}_{X}\left(E_{a}+F_{a}\right)$, for $a \in A_{n}$, so the above corollary gives us a normalized block $z \in Z^{\prime}$ such that for every $a \in A$ the pair $\left(E_{a}+\langle z\rangle, F_{a}\right)$ rejects $Z_{0}$. The pair $\left(F_{a}, E_{a}\right)$ is also on the list, thus the pair $\left(F_{a}+\langle z\rangle, E_{a}\right)$ rejects the space $Z_{0}$ as well. Then we take $z_{n+1}=z$. It is clear now that for that choice the pair $\left(E_{I}, E_{J}\right)$ rejects the space $Z_{0}$ for every partition $I \sqcup J=\{1, \ldots, n+1\}$.

We will show now that the space $Y=\left\langle z_{n} \mid n \in \mathbb{N}\right\rangle$ is $\mathcal{F}_{\alpha}$-asymptotically unconditional with constant $\frac{4}{\varepsilon}$. Indeed, take $\left(x_{1}, \ldots, x_{k}\right) \in \Sigma_{\mathcal{F}_{\alpha}}(Y) \subset \Sigma_{\mathcal{F}_{\alpha}}(X)$. Then there exists $n$ such that $\left(x_{1}, \ldots, x_{k}\right) \in\left\langle z_{1}, \ldots, z_{n}\right\rangle$. Our final step is to show that for every partition $I_{0} \sqcup J_{0}=\{1, \ldots, k\}$ we have $b\left(\left\langle x_{i} \mid i \in I_{0}\right\rangle,\left\langle x_{j} \mid j \in J_{0}\right\rangle\right) \geq \frac{\varepsilon}{2}$ which combined with Proposition 2.14 will finish the proof of the theorem.

Fix a partition $I_{0} \sqcup J_{0}=\{1, \ldots, k\}$ and let $I \sqcup J=\{1, \ldots, n\}$ be the respective partition for $\left(z_{1}, \ldots, z_{n}\right)$. We then have $\left(x_{i}\right)_{i \in I_{0}} \in \Sigma\left(E_{I}\right)$ and $\left(x_{j}\right)_{j \in J_{0}} \in \Sigma\left(E_{J}\right)$. Finally

$$
\begin{aligned}
b\left(\left\langle x_{i} \mid i \in I_{0}\right\rangle,\left\langle x_{j} \mid j \in J_{0}\right\rangle\right) & \geq \frac{1}{2} a\left(\left\langle x_{i} \mid i \in I_{0}\right\rangle,\left\langle x_{j} \mid j \in J_{0}\right\rangle\right) \\
& \geq \frac{1}{2} k_{\mathcal{F}_{\alpha}}\left(E_{I}, E_{J}\right) \geq \frac{\varepsilon}{2} .
\end{aligned}
$$

\subsection{Main ingredients}

Before we proceed to the proofs of the main theorems we need two more lemmas. The first one is a simple geometric one concerning the influence of cutting off initial vectors on the angle between generated subspaces. The second one is combinatorial and provides a way of passing from $\mathcal{F}_{\alpha}$-admissible vectors in $X$ to $\mathcal{F}_{\beta}$-admissible vectors in $Y$ for $\alpha<\beta$.

Lemma 4.11 If $\varepsilon<\frac{1}{2}$ and a sequence $\left(u_{i}\right)_{i=0}^{K} \in \Sigma(X)$ satisfy $b\left(\left\langle u_{2 i}\right| i \geq\right.$ $\left.0\rangle,\left\langle u_{2 i+1} \mid i \geq 0\right\rangle\right)<\varepsilon$, then $K \geq 3$ and $b\left(\left\langle u_{2 i} \mid i \geq 1\right\rangle,\left\langle u_{2 i+1} \mid i \geq 1\right\rangle\right)<4 \varepsilon$.

Lemma 4.12 If $\alpha<\beta<\omega_{1}$ and $X$ is a Banach space with a bimonotone basis and $W \subset X$ is a block subspace with a basis $\left(e_{n}\right)_{n \in \mathbb{N}}$, then 
1. there exists a set $M \in[\mathbb{N}]$ such that for the space $W_{0}=\left\langle e_{m} \mid m \in M\right\rangle$ the following inclusion holds

$$
\Sigma_{\mathcal{F}_{\alpha}}(X) \cap \Sigma\left(W_{0}\right) \subset \Sigma_{\mathcal{F}_{\beta}}(W)
$$

2. there exists a set $M^{\prime} \in[\mathbb{N}]$ such that card $\left(M^{\prime} \cap(2 \mathbb{N}+j)\right)=\aleph_{0}$, for $j=0,1$, and the space $W_{0}^{\prime}=\left\langle e_{m^{\prime}} \mid m^{\prime} \in M^{\prime}\right\rangle$ satisfies:

$$
\forall l \geq 2 \quad \forall\left(w_{0}, \ldots, w_{l}\right) \in \Sigma_{\mathcal{F}_{\alpha}}(X) \cap \Sigma\left(W_{0}^{\prime}\right):\left(w_{2}, \ldots, w_{l}\right) \in \Sigma_{\mathcal{F}_{\beta}}(W)
$$

Proof For a set $\Phi \subset \Sigma(X)$ we define

$\mathcal{F}_{X}(\Phi)=\left\{\left(n_{1}, \ldots, n_{k}\right) \mid \exists\left(x_{1}, \ldots, x_{k}\right) \in \Phi: n_{i}=\min \left(\operatorname{supp}_{X} x_{i}\right)\right.$ for $\left.i=0, \ldots, k\right\}$

1. We use Gasparis' theorem (Theorem 2.4) for $\mathcal{F}=\mathcal{F}_{W}\left(\Sigma_{\mathcal{F}_{\alpha}}(X) \cap \Sigma(W)\right), \mathcal{G}=$ $\mathcal{F}_{W}\left(\Sigma_{\mathcal{F}_{\beta}}(W)\right), N=\mathbb{N}$. We obtain a set $M \in[N]$ such that

$$
\begin{aligned}
\mathcal{F}_{W}\left(\Sigma_{\mathcal{F}_{\alpha}}(X) \cap \Sigma(W)\right)[M] & \subset \mathcal{F}_{W}\left(\Sigma_{\mathcal{F}_{\beta}}(W)\right) \text { or } \\
\mathcal{F}_{W}\left(\Sigma_{\mathcal{F}_{\beta}}(W)\right)[M] & \subset \mathcal{F}_{W}\left(\Sigma_{\mathcal{F}_{\alpha}}(X) \cap \Sigma(W)\right) .
\end{aligned}
$$

The second inclusion is impossible due to $\beta>\alpha$, since, based on axiom $(\mathcal{F} 3)$, we have $I\left(\mathcal{F}_{W}\left(\Sigma_{\mathcal{F}_{\beta}}(W)\right)[M]\right)>I\left(\mathcal{F}_{W}\left(\Sigma_{\mathcal{F}_{\alpha}}(X) \cap \Sigma(W)\right)\right)$.

For the basis $\left(e_{n}\right)_{n \in \mathbb{N}}$ of $W$ denote $W_{0}=\left\langle e_{m} \mid m \in M\right\rangle$ and fix $\left(v_{1}, \ldots, v_{k}\right) \in$ $\Sigma_{\mathcal{F}_{\alpha}}(X) \cap \Sigma\left(W_{0}\right)$. Then $\left(n_{1}, \ldots, n_{k}\right) \in \mathcal{F}_{W}\left(\Sigma_{\mathcal{F}_{\alpha}}(X) \cap \Sigma(W)\right)[M] \subset \mathcal{F}_{W}$ $\left(\Sigma_{\mathcal{F}_{\beta}}(W)\right)$, where $n_{i}=\min \left(\operatorname{supp}_{W}\left(v_{i}\right)\right)$, hence we conclude that $\left(v_{1}, \ldots, v_{k}\right) \in$ $\Sigma_{\mathcal{F}_{\beta}}(W)$.

2. Let $M$ and $W_{0}$ be as in (1). If $\operatorname{card}(M \cap(2 \mathbb{N}+j))=\aleph_{0}$, for $j=0,1$ then $M$ and $W_{0}$ are the required spaces and we are done. On the other hand we have $\operatorname{card}(M \cap 2 \mathbb{N})=\aleph_{0} \operatorname{or} \operatorname{card}(M \cap(2 \mathbb{N}+1))=\aleph_{0}$, so suppose that $\operatorname{card}(M \cap 2 \mathbb{N})=$ $\aleph_{0}$ and $\operatorname{card}(M \cap(2 \mathbb{N}+1))<\aleph_{0}$. The proof in the second case is analogous.

Let us define an injection $\psi: M \hookrightarrow \mathbb{N}$ by the formula $\psi(4 m+2 j)=4 m+j, 4 m+$ $2 j \in M, j=0,1$. Then we set $M^{\prime}=\psi(M)$ and $W_{0}^{\prime}=\left\langle x_{m}^{\prime} \mid m^{\prime} \in M^{\prime}\right\rangle$.

Fix $l \geq 2$ and $\left(w_{0}, \ldots, w_{l}\right) \in \Sigma_{\mathcal{F}_{\alpha}}(X) \cap \Sigma\left(W_{0}^{\prime}\right)$ and denote $n_{i}=\min \left(\operatorname{supp}_{W}\left(w_{i}\right)\right)$ for $i=0, \ldots, l$. Then we obtain $\left(n_{0}, \ldots, n_{l}\right) \in \mathcal{F}_{W}\left(\Sigma_{\mathcal{F}_{\alpha}}(X) \cap \Sigma(W)\right)\left[M^{\prime}\right]$. The set $\mathcal{F}_{W}\left(\Sigma_{\mathcal{F}_{\alpha}}(X) \cap \Sigma(W)\right)$ is spreading so $\left(\psi^{-1}\left(n_{0}\right), \ldots, \psi^{-1}\left(n_{l}\right)\right)$ lives in $\mathcal{F}_{W}\left(\Sigma_{\mathcal{F}_{\alpha}}(X) \cap\right.$ $\Sigma(W))[M]$, hence the definition of $M$ gives us that

$$
\left(\psi^{-1}\left(n_{0}\right), \ldots, \psi^{-1}\left(n_{l}\right)\right) \in \mathcal{F}_{W}\left(\Sigma_{\mathcal{F}_{\beta}}(W)\right)
$$

Moreover, $n_{i}>n_{i-1}$ and $n_{i-1}+1 \geq \psi^{-1}\left(n_{i-1}\right)$, which implies the inequality $n_{i}>\psi^{-1}\left(n_{i-2}\right)$, for $i=2, \ldots, l$. Therefore the regularity of the set $\mathcal{F}_{W}\left(\Sigma_{\mathcal{F}_{\beta}}(W)\right)$ gives us $\left(n_{2}, \ldots, n_{l}\right) \in \mathcal{F}_{W}\left(\Sigma_{\mathcal{F}_{\beta}}(W)\right)$, hence $\left(w_{2}, \ldots, w_{l}\right) \in \Sigma_{\mathcal{F}_{\beta}}(W)$.

We will use the above lemma in the proof of the following 
Proposition 4.13 Let $X$ be a Banach space with a basis, let $Y$ be an infinite dimensional block subspace of $X$, let $\alpha<\beta$ be countable ordinals and let $\varepsilon>0$. Then, if the subspace $Y$ has the $H I_{\mathcal{F}_{\alpha}, X}(\varepsilon)$ property, then $Y$ has the $H I_{\mathcal{F}_{\beta}}(4 \varepsilon)$ property.

Proof Let us fix infinite dimensional subspaces $U_{0}, U_{1} \subset Y$. We will construct a sequence $\left(u_{i}\right)_{i}$ which satisfies the following conditions:

1. $\left(u_{i}\right)_{i} \in \Sigma_{\mathcal{F}_{\beta}}(Y)$ and $\left(u_{2 i+j}\right)_{i} \in \Sigma\left(U_{j}^{\prime}\right), j=0,1$,

2. $b\left(\left\langle u_{2 i}\right\rangle,\left\langle u_{2 i+1}\right\rangle\right)<4 \varepsilon$.

In order to do that we pick inductively a block sequence $\left(x_{n}\right)_{n=0}^{\infty}$ satisfying: $x_{2 k+j} \in$ $U_{j}, j=0,1, k \in \mathbb{N}$. Then we set $W=\left\langle x_{k} \mid k \in \mathbb{N}\right\rangle$. Of course we have $W \subset Y$. Using the first part of Lemma 4.12 we obtain a set $M \in[\mathbb{N}]$ and a subspace $W_{0}=$ $\left\langle x_{m} \mid m \in M\right\rangle \subset W$ such that:

$$
\Sigma_{\mathcal{F}_{\alpha}}(X) \cap \Sigma\left(W_{0}\right) \subset \Sigma_{\mathcal{F}_{\beta}}(W) \subset \Sigma_{\mathcal{F}_{\beta}}(Y)
$$

Case $I \operatorname{card}(M \cap(2 \mathbb{N}+j))=\aleph_{0}$ for $j=0,1$.

Set $U_{j}^{\prime}=U_{j} \cap W_{0}$. Then we have $\operatorname{dim} U_{j}^{\prime}=\infty$. Using the definition of the $H I_{\mathcal{F}_{\alpha}, X}(\varepsilon)$ property, for the pair $\left(U_{0}^{\prime}, U_{1}^{\prime}\right)$ we obtain a sequence $\left(u_{i}\right)_{i=0}^{K}$ satisfying:

(i) $\left(u_{i}\right)_{i=0}^{K} \in \Sigma_{\mathcal{F}_{\alpha}}(X)$ and $\left(u_{2 i+j}\right)_{i} \in \Sigma\left(U_{j}^{\prime}\right), j=0,1$,

(ii) $b\left(\left\langle u_{2 i}\right\rangle,\left\langle u_{2 i+1}\right\rangle\right)<\varepsilon<4 \varepsilon$.

Using (†) and property (i) we conclude that: $\left(u_{i}\right)_{i} \in \Sigma_{\mathcal{F}_{\alpha}}(X) \cap \Sigma\left(W_{0}\right) \subset \Sigma_{\mathcal{F}_{\beta}}(Y)$.

Case II $\operatorname{card}(M \cap(2 \mathbb{N}+j))<\aleph_{0}$ for some $j$.

The second part of Lemma 4.12 allow us to improve the set $M$ to obtain a set $M^{\prime}$ like in case I and a subspace $W_{0}^{\prime}=\left\langle x_{m} \mid m \in M^{\prime}\right\rangle$ with the following property:

$$
\forall l \geq 2 \quad \forall\left(w_{0}, \ldots, w_{l}\right) \in \Sigma_{\mathcal{F}_{\alpha}}(X) \cap \Sigma\left(W_{0}^{\prime}\right):\left(w_{2}, \ldots, w_{l}\right) \in \Sigma_{\mathcal{F}_{\beta}}(W) .
$$

If we set $U_{j}^{\prime \prime}=U_{j} \cap W_{0}^{\prime}, j=0,1$, then $\operatorname{dim} U_{j}^{\prime \prime}=\infty, j=0,1$.

Hereafter, just as in case I we use the definition of the $H I_{\mathcal{F}_{\alpha}, X}(\varepsilon)$ property for the pair $\left(U_{0}^{\prime}, U_{1}^{\prime}\right)$ which gives us a sequence $\left(u_{i}\right)_{i=0}^{K}$ satisfying:

(i') $\left(u_{i}\right)_{i=0}^{K} \in \Sigma_{\mathcal{F}_{\alpha}}(X):\left(u_{2 i+j}\right)_{i} \in \Sigma\left(U_{j}^{\prime \prime}\right), j=0,1$,

(ii') $b\left(\left\langle u_{2 i} \mid i \geq 0\right\rangle,\left\langle u_{2 i+1} \mid i \geq 0\right\rangle\right)<\varepsilon$.

Using ( $\ddagger$ ) and (i') we have: $\left(u_{i}\right)_{i=2}^{K} \in \Sigma_{\mathcal{F}_{\beta}}(Y)$. Using now property (ii') and Lemma 4.11 we have: $b\left(\left\langle u_{2 i} \mid i \geq 1\right\rangle,\left\langle u_{2 i+1} \mid i \geq 1\right\rangle\right)<4 \varepsilon$.

\subsection{Proof of Wagner's theorem [version $\mathcal{F}$ ]}

Let us fix a subspace $Z_{0} \subset X$ for which the following ordinal number

$$
\beta\left(Z_{0}\right)=\min \left\{\gamma<\omega_{1} \mid \neg\left(\exists W \subset Z_{0}: W \text { is } \mathcal{F}_{\gamma} \text {-asymptotically unconditional }\right)\right\}
$$

attains the minimum in the definition of the ordinal number $\beta=\min \{\beta(Z) \mid Z<X\}$. Then for every subspace $Y$ of the space $Z_{0}$ we have $\beta(Y)=\beta$. Therefore any subspace 
$Y$ of $Z_{0}$ satisfies property (i) of the theorem. Hence, without loss of generality we can assume $X=Z_{0}$. The proof is divided into three steps. In the first one we show some property of the space $X$ which will allow us to construct the desired subspace $Y$. The main ingredient is the quantified version of the $\mathcal{F}_{\alpha}$-dichotomy (Theorem 4.1).

Step 1 For every $\varepsilon>0, Z \subset X$ there exist a subspace $W \subset Z$ and an ordinal $\alpha<\beta$ such that the subspace $W$ is $H I_{\mathcal{F}_{\alpha}, X}(\varepsilon)$, i.e. the following holds:

$\forall U_{0}, U_{1} \subset W \exists\left(u_{i}\right)_{i=0}^{K} \in \Sigma_{\mathcal{F}_{\alpha}}(X):\left(u_{2 i+j}\right)_{i} \in \Sigma\left(U_{j}\right)$ and $b\left(\left\langle u_{2 i}\right\rangle,\left\langle u_{2 i+1}\right\rangle\right)<\varepsilon$

Let us assume the contrary:

$$
\exists \varepsilon>0 \exists \widehat{Z} \subset X \forall W \subset \widehat{Z} \forall \alpha<\beta: W \text { is not } H I_{\mathcal{F}_{\alpha}, X}(\varepsilon) .
$$

Fix a subspace $W \subset \widehat{Z}$ and an ordinal $\alpha<\beta$. Theorem 4.1 implies the existence in the subspace $W$ of a further subspace $W_{\alpha}$ which is $\mathcal{F}_{\alpha}$-asymptotically unconditional with constant $4 / \varepsilon$. We have just showed that

$$
\forall \alpha<\beta \quad \forall W \subset \widehat{Z} \exists W_{\alpha} \subset W: W_{\alpha} \text { is } \mathcal{F}_{\alpha} \text {-asymptotically unc. with constant } \frac{4}{\varepsilon} \text {. }
$$

Let $\left(\beta_{n}\right)$ be the sequence of ordinals fixed in the definition of the family $\mathcal{F}_{\beta}$. Using the above property we construct a decreasing sequence of subspaces $\left(W_{n}\right)_{n=1}^{\infty}$ such that for every positive integer $n$ the subspace $W_{n}$ is $\mathcal{F}_{\beta_{n}}$-asymptotically unconditional with constant $4 / \varepsilon$.

Then we construct inductively a block sequence $\left(w_{n}\right)_{n \in \mathbb{N}}$ and we set a diagonal space of the sequence $\left(W_{n}\right)$ to be $\widehat{W}=\left\langle w_{n} \mid n \in \mathbb{N}\right\rangle$ which due to the uniformly bounded asymptotically unconditional constants of the spaces $\left\{W_{n}\right\}$ is $\mathcal{F}_{\beta}$-asymptotically unconditional. Indeed, fix a sequence $\left(x_{1}, \ldots, x_{k}\right) \in \Sigma_{\mathcal{F}_{\beta}}(\widehat{W})$. Then there exists $n \in \mathbb{N}$ such that the sequence $\left(x_{1}, \ldots, x_{k}\right) \in \Sigma_{\mathcal{F}_{\beta_{n}}}(\widehat{W})$ and $\min \left(\operatorname{supp}_{\widehat{W}} x_{1}\right) \geq n$. From the definition of a diagonal subspace we have that $\Sigma_{\mathcal{F}_{\beta_{n}}}(\widehat{W}) \cap \Sigma\left([\widehat{W}]_{n}\right) \subset \Sigma_{\mathcal{F}_{\beta_{n}}}\left(W_{n}\right)$, where $[\widehat{W}]_{n}=\left\{x \in \widehat{W} \mid \min \left(\operatorname{supp}_{\widehat{W}} x\right) \geq n\right\} \subset$ $W_{n}$, hence the sequence $\left(x_{1}, \ldots, x_{k}\right)$ belongs to the set $\Sigma_{\mathcal{F}_{\beta_{n}}}\left(W_{n}\right)$. Thus we obtain that the sequence $\left(x_{1}, \ldots, x_{k}\right)$ is $4 / \varepsilon$-unconditional.

Thus we have constructed the space which is $\mathcal{F}_{\beta}$-asymptotically unconditional which is contradictory with the choice of the ordinal $\beta$. The proof of step 1 is completed.

In the next step we will construct a subspace $Y$ and a sequence $\left(\alpha_{n}\right)$ stabilizing the above property of the space $X$ with respect to $\varepsilon$. Then in the final step we will show that it is enough to renumber the sequence $\left(\alpha_{n}\right)$ in order to obtain property (2) of the theorem.

Step 2 There exist a sequence $\alpha_{n} \nearrow \beta$ and a space $Y$ which has the $H I_{\mathcal{F}_{\alpha_{n}}, X}\left(\frac{1}{n}\right)$ property for every positive integer $n$, i.e. the following condition holds:

$$
\forall U_{0}, U_{1} \subset Y \exists\left(u_{i}^{n}\right)_{i=0}^{K_{n}} \in \Sigma_{\mathcal{F}_{\alpha_{n}}}(X):\left(u_{2 i+j}^{n}\right)_{i} \in \Sigma\left(U_{j}\right) \text { and } b\left(\left\langle u_{2 i}^{n}\right\rangle,\left\langle u_{2 i+1}^{n}\right\rangle\right)<\frac{1}{n} .
$$


The above property is weaker than the desired one because we demand the testing sequence $\left(u_{i}^{n}\right)$ to be admissible only in the super-space $X$.

Using step 1 we construct inductively a sequence $\left(\alpha_{n}\right)$ and a decreasing sequence of subspaces $\left(Z_{n}\right)$ such that each space $Z_{n}$ is $H I_{\mathcal{F}_{\alpha_{n}}, X}\left(\frac{1}{n}\right)$. Then we construct the diagonal space $Y$ out of the spaces $\left(Z_{n}\right)$. The space $Y$ is contained in the space $Z_{n}$ up to a finite dimension, so that the space $Y$ is $H I_{\mathcal{F}_{\alpha_{n}}, X}\left(\frac{1}{n}\right)$ for every positive integer $n$.

It remains to show that $\alpha_{n} \nearrow \beta$. Let us assume the contrary, i.e. there exists an ordinal $\gamma$ such that for every $n$ we have: $\alpha_{n}<\gamma<\beta$. By the definition of $\beta$ we have a subspace $\widehat{Y} \subset Y$ which is $\mathcal{F}_{\gamma}$-asymptotically unconditional with constant $D_{\gamma}$. Let us fix $N \geq D_{\gamma}$. Using part 1 of Lemma 4.12 we obtain a subspace $Y_{0} \subset \widehat{Y}$ such that $\Sigma_{\mathcal{F}_{\alpha_{N}}}(X) \cap \Sigma\left(Y_{0}\right) \subset \Sigma_{\mathcal{F}_{\gamma}}(\widehat{Y})$.

Using the $H I_{\mathcal{F}_{\alpha_{N}}, X}\left(\frac{1}{N}\right)$ property for the subspaces $U_{0}, U_{1}=Y_{0}$, we obtain a sequence $\left(u_{i}\right) \in \Sigma_{\mathcal{F}_{\alpha_{N}}}(X)$ satisfying the following conditions:

1. $\left(u_{2 i+j}\right) \in \Sigma\left(Y_{0}\right), j=0,1$,

2. $b\left(\left\langle u_{2 i}\right\rangle,\left\langle u_{2 i+1}\right\rangle\right)<\frac{1}{N}$.

Condition (1) implies $\left(u_{i}\right) \in \Sigma_{\mathcal{F}_{\alpha_{N}}}(X) \cap \Sigma\left(Y_{0}\right)$, so $\left(u_{i}\right) \in \Sigma_{\mathcal{F}_{\gamma}}(\widehat{Y})$, hence the sequence $\left(u_{i}\right)$ has unconditional constant less then or equal to $D_{\gamma}$.

On the other hand condition (2) and Proposition 2.14 imply that the sequence $\left(u_{i}\right)$ has unconditional constant greater than $N \geq D_{\gamma}$, which yields a contradiction. Hence $\sup _{n} \alpha_{n}=\beta$ and passing to a subsequence we can assume that $\left(\alpha_{n}\right)_{n \in \mathbb{N}}$ is increasing. The proof of step 2 is complete.

Step 3 There exist a subspace $Y \subset X$ and a sequence $\alpha_{n}^{\prime} \nearrow \beta$ such that the subspace $Y$ is $H I_{\mathcal{F}_{\alpha_{n}^{\prime}}}\left(\frac{1}{n}\right)$ for every $n \in \mathbb{N}$, i.e.

$\forall U_{0}, U_{1} \subset Y \exists\left(u_{i}^{n}\right)_{i=0}^{K_{n}} \in \Sigma_{\mathcal{F}_{\alpha_{n}}}(Y):\left(u_{2 i+j}^{n}\right)_{i} \in \Sigma\left(U_{j}\right)$ and $b\left(\left\langle u_{2 i}^{n}\right\rangle,\left\langle u_{2 i+1}^{n}\right\rangle\right)<\frac{1}{n}$.

Using Proposition 4.13 with $\alpha=\alpha_{4 n}, \beta=\alpha_{4 n+1}$ for every positive integer $n$ we obtain that the subspace $Y$ constructed in step 2 and the sequence consisting of elements $\alpha_{n}^{\prime}=\alpha_{4 n+1}, n \in \mathbb{N}$ satisfy the requirements.

The proof of Wagner's theorem [version $\mathcal{F}$ ] is finished.

\subsection{Proof of the $\mathcal{F}_{\alpha}$-dichotomy}

We give the proofs of $\mathcal{F}_{\alpha}$-dichotomy and its version for limit ordinals.

Proof of the $\mathcal{F}_{\alpha}$-dichotomy (Theorem 3.4) Let $X$ be a Banach space with a basis and without an unconditional sequence and let $\alpha$ be a countable ordinal number. If there exists a subspace which is $\mathcal{F}_{\alpha}$-asymptotically unconditional then we are done. Suppose then such a space does not exist. Set $Y_{0}=X$ and construct inductively a decreasing sequence of subspaces $\left(Y_{n}\right)_{n \in \mathbb{N}}$ such that for every $n$ the space $Y_{n}$ has the $H I_{\mathcal{F}_{\alpha}, X}\left(\frac{1}{n}\right)$ property. For fixed $n>0$ using the quantified version of the $\mathcal{F}_{\alpha}$-dichotomy we obtain that in the space $Y_{n-1}$ there exists a subspace $Y_{n}$ which is $H I_{\mathcal{F}_{\alpha}, X}\left(\frac{1}{n}\right)$.

Let $Y$ be a diagonal space of the sequence $\left(Y_{n}\right)_{n \in \mathbb{N}}$. Then the space $Y$ is $H I_{\mathcal{F}_{\alpha}, X}\left(\frac{1}{n}\right)$ for every $n \in \mathbb{N}$. 
Now using Proposition 4.13 we conclude that the space $Y$ is $H I_{\mathcal{F}_{\alpha+1}}\left(\frac{1}{n}\right)$ for every positive integer $n$. Hence, the space $Y$ is $H I_{\mathcal{F}_{\alpha+1}}$, which finishes the proof.

Now using Wagner's theorem [version $\mathcal{F}$ ] we can strengthen the conclusion of the $\mathcal{F}_{\alpha}$-dichotomy.

Proof of the $\mathcal{F}_{\alpha}$-dichotomy for limit ordinals Let $X$ be a Banach space with a basis and without an unconditional sequence and let $\alpha$ be a countable limit ordinal number. We define the ordinal $\beta$ like in Wagner's theorem as $\beta=\min \{\beta(Z) \mid Z \subset X\}$.

There are three cases:

1. If $\alpha<\beta$, then the definition of $\beta$ implies that in the space $X$ there exists a subspace $Y$ which is $\mathcal{F}_{\alpha}$-asymptotically unconditional and we are through.

2. If $\alpha=\beta$, then the assumptions of Wagner's theorem (Theorem 3.2) are satisfied. Therefore we obtain a sequence $\alpha_{n} \nearrow \beta$ and a subspace $Y$ which is $H I_{\mathcal{F}_{\alpha_{n}}}\left(\frac{1}{n}\right)$ for every positive integer $n$. Using again Proposition 4.13 we conclude that $Y$ is $H I_{\mathcal{F}_{\alpha}}$.

3. If $\alpha>\beta$, then the definition of $\beta$ implies that in the space $X$ there is no subspace which is $\mathcal{F}_{\beta}$-asymptotically unconditional. As in the previous proof of the $\mathcal{F}_{\alpha}$-dichotomy using the quantified version of the $\mathcal{F}_{\alpha}$-dichotomy and a diagonal subspace we can construct a subspace $Y$ which is $H I_{\mathcal{F}_{\beta+1}}$. Thus Proposition 4.13 implies that the space $Y$ has the $H I_{\mathcal{F}_{\alpha}}$ property.

Open Access This article is distributed under the terms of the Creative Commons Attribution 4.0 International License (http://creativecommons.org/licenses/by/4.0/), which permits unrestricted use, distribution, and reproduction in any medium, provided you give appropriate credit to the original author(s) and the source, provide a link to the Creative Commons license, and indicate if changes were made.

\section{References}

1. Maurey, B., Gowers, W.: The unconditional basic sequence problem. J. Am. Math. Soc. 6, 851-874 (1993)

2. Maurey, B., Milman, V., Tomczak-Jaegermann, N.: Infinite dimensional asymptotic theory for Banach spaces. In: GAFA, Op. Theory: Adv. and App., vol. 77, pp. 149-175. Birkhauser, Basel (1995)

3. Wagner, R.: Gowers' dichotomy for asymptotic structure. Proc. Am. Math. Soc. 124, 3089-3096 (1996)

4. Tomczak-Jaegermann, N.: Banach spaces of type $p$ have arbitrarily distortable subspaces. GAFA 6, 1074-1082 (1996)

5. Wagner, R.: Finite high-order games and an inductive approach towards Gowers' dichotomy. Ann. Pure Appl. Log. 111, 39-60 (2001)

6. Argyros, S., Tolias, A.: Methods in the theory of hereditarily indecomposable Banach spaces. In: Mem. of the Am. Math. Soc., vol. 806, pp. 1-113 (2004)

7. Gasparis, I.: A dichotomy theorem for subsets of the power set of the natural numbers. Proc. Am. Math. Soc. 129, 759-764 (2000)

8. Alspach, D., Argyros, S.: Complexity of weakly null sequences. Diss. Math. 321, 1-44 (1992)

9. Odell, E., Tomczak-Jaegermann, N., Wagner, R.: Proximity to $\ell_{1}$ spaces. J. Funct. Anal. 150, 101-145 (1997)

10. Argyros, S., Deliyanni, I.: Examples of asymptotic- $\ell_{1}$ Banach spaces. Trans. Am. Math. Soc. 3, $973-$ 995 (1997)

11. Maurey, B.: A note on Gowers' dichotomy theorem. Convex Geom. Anal. 34, 149-157 (1998)

12. Milman, V., Sharir, M.: Shrinking minimal systems and compementation of $\ell_{p}^{n}$-spaces in reflexive Banach spaces. Proc. Lond. Math. Soc. 39, 1-29 (1979)

13. Gowers, W.T.: A hereditarily indecomposable space with an asymptotic unconditional basis. In: GAFA, Op. Theory: Adv. and App., vol. 77, pp. pp. 111-120. Birkhauser, Basel (1995) 
14. Gowers, W.T.: An infinite Ramsey theorem and some Banach-space dychotomies. Ann. Math. 153, 797-833 (2002)

15. Galvin, F., Prikry, K.: Borel sets and Ramsey’s theorem. J. Symb. Log. 38, 193-198 (1973) 